\title{
The Intake of Aronia Melanocarpa Fruits and Juice has Great Potential for Prevention of Socially Significant Diseases
}

\author{
Margaritka Boncheva* \\ Clinical Laboratory, Ekaterina University Hospital, Bulgaria
}

*Corresponding author: Margaritka Boncheva, Clinical Laboratory, Ekaterina University Hospital, Professor at the Medical University of Varna, Bulgaria.

To Cite This Article: Margaritka Boncheva. The Intake of Aronia Melanocarpa Fruits and Juice has Great Potential for Prevention of Socially Significant Diseases. Am J Biomed Sci \& Res. 2019 - 5(3). AJBSR.MS.ID.000910. DOI: 10.34297/AJBSR.2019.05.000910.

Received: 眥 September 14, 2019; Published: 眥 September 23, 2019

Keywords: Aronia melanocarpa; Antioxidant capacity; Prevention of diseases

\section{Introduction}

Socially significant diseases are preceded by changes in metabolism, oxidative stress and changes in the vascular wall of the arteries leading to poor blood supply to the organs, insufficient oxygen to the organs or thrombosis. Prevention of these diseases involves clarifying genetic predispositions and changing lifestyles (diet and physical activity). Nutrition is a major social problem in the presence of a lot of aggression in the food industry and the supply of very low-quality food. The homeland of Aronia bushes is North America. The fruits of this plant have been known for their medicinal properties since ancient times. Today, we rediscover this fruit as a true, useful food, which has, so far, been only incidentally sought in states of disease coming close to the incurable ones. The current research points to the necessity of including this food in our daily diet as a grace to the healthy lifestyle. Epidemiological studies and associated meta-analyses strongly suggest that long-term diets rich in plant polyphenols offer protection against development of cancers, cardiovascular disease, diabetes, osteoporosis and neurodegenerative diseases. The aronia fruit can be consumed as fresh fruit, cold pressed juice from fresh fruits or the so-called "fruit" wine, dried (in the form of flour) or frozen [1,2].

Fruits are important dietary sources of polyphenols. Main polyphenols determined by different assays are: Cyanidin-3galactoside, Cyanidin-3-arabinoside, Cyanidin-3-glucoside, Cyanidin-3-xyloside, Quercetin, Quercetin-galactoside, Quercetinglucoside, Chlorogenic acide, ß-carotens. Aronia contains 5 times the amounts of these substances as compared to grapes, grapefruit, and the beetroot! Remarkable presence of minerals and microelements: iodine, potassium, calcium, phosphorus, magnesium, and iron. Record concentrations of iodine and manganese. Vitamins: A, B1, B2, B3, B5, B6, B9, C, E, K, P, traces of B17. Small quantities of fructose required to satisfy daily needs; sorbitol and parasorbid, which attribute some weak to moderate laxative effects to the drink. It contains the adequate concentrations of the essential amino acids - arginine, lysine, histidine, threonine; as well as of the non-essential amino acids - tyrosine, cysteine, L-alanine, aspartic acid, serine, glutamine. In 2015, Naman and al isolated from dried aronia melanocarpa fruit, Melanodiol4-0protocatechuate and melanodiol, which represent novel flavonoid derivatives. This is an unprecedented pair of natural products derivates of flavonoids containing a fused pentacyclic core with two contiguous hemiketals.

Each compound demonstrated potent hydroxyl radical scavenging activity, moderate quinone reductase-inducing activity, and no notable cytotoxicity to murine hepa1c1c7 cells in vitro. These non-crystalline molecules were isolated from aronia melanocarpa and significant challenges in their structural determination were overcome using computer-assisted structure elucidation software [2-4]. The numerous healing effects are based on the universal antioxidant capacity of the ingredients. Scientifically validated data have been published to report an improvement in patients' oxidative status through Carrateli panel testing: SHp Test, d-ROMs Test, OXY-Adsorbent Test, LP CHOLOX Test, Anti-ROMs Test, BAP Test, ROSA Test. Thus, the antioxidant effect of aronia intake in humans has been demonstrated by three different mechanisms. A daily supplement of aronia juice to one's routine diet regulates the quantity of oxidative active radicals - free radicals (ROS). Alongside, the antioxidant capacity of blood plasma is improved [5-10]. 
Considerable influence on erythrocyte membrane and hemoglobin function is discussed due to the proven antioxidant effects of the polyphenols contained in the fruits of the melanocarpa aronia and the rich content of minerals, vitamins and trace elements [11-15] Aronia berries have a beneficial effect on liver fibrosis. The recovery from liver fibrosis is associated with expression levels of Gadd45g and Igfbp1 in the liver. The beneficial effects of aronia berries on liver fibrosis reduce the risk of liver cancer diseases and insulin resistance, resulting in reduction of serum FABP1 and FABP4 levels.

Further study is needed to identify the compound(s) in aronia berries that is effective against liver fibrosis through regulation of Gadd45g and Igfbp1 expression [2,12,13]. Beneficial effects on type 2 diabetes have been reported in many scientific publications [16-18]. Pathobiochemical mechanisms of diabetes mellitus based on insulin resistance are favorably affected by aronia juice intake $[2,6,14]$. Yamane et al. [10-12] reported that DPP IV activity is inhibited by aronia juice. DPP IV inhibitor is cyanidin 3, 5-diglucoside and $\alpha$-glucosidase in aronia juice. DPP IV is inhibited by cyanidin 3,5-diglucoside more than cyanidin and cyanidin 3-glucoside $[10,15,16]$. Hypertension is improved and medication intake is reduced after the use of chokeberry juice [17]. Aronia berries inhibit ACE activity in the kidney and reduction of ACE activity in the local renin-angiotensin system. Aronia berries may also become a useful drug for the prevention of hypotension $[11,19]$. A multifaceted healing effect has been manifested with models of diseases after the course of aronia juice intake, for instance: a general anti-inflammatory effect, regardless of the process localization; an antimutagenic effect; facilitating heart activity by stabilizing the heart muscle, the circulation system and enhancing the blood supply through strengthening the blood vessels of the heart (due to removal of atheromatous plaques and endothelial dysfunction). Aronia intake also:

a) Prevents the development of or improves hypertonic disease by restoring the elasticity of the arterial blood vessels;

b) Improves the hepatic function by facilitating metabolic processes in the liver cells (less "poisons" reach the liver);

c) Reconstructs the mucosa in gastro-intestinal tract in cases where it has been impaired;

d) Acts directly on various pathogenic microorganisms viruses and bacteria, and stimulates immune-active cells to cope with infections;

e) When exposing animals to radiation, those who have taken aronia fruit juice show a bigger number and a higher activity of CD4 and CD8 lymphocytes (the cells fighting the destructive effects of radiation);

f) Aronia products improve glucose assimilation by the cells (intracellular transportation of glucose) in patterns of Type 2 diabetes mellitus (T2DM) whereby the values of blood glucose are reduced and the patients' condition is favorably affected; other mechanisms of influencing T2DM are currently being investigated.

g) It has been established that aronia products reduce thrombocyte aggregation and prevent thrombi formation in the arteries.

Last, but not least, we should mention the favorable neuropsychological effects experienced by subjects in the groups under study [20-24]. Clinical investigations with people are a bit more limited; however, they undoubtedly supplement and by no means contradict the results reported from the experiments with animals and cellular culture patterns. It can definitely be concluded that the consumption of aronia juice does not have any toxic effects! Eventual over-dosage may have a laxative effect, which is terminated through dosage reduction, and this is individually conditioned. This publication reviewed the scientific research regarding the health benefits of black chokeberry fruits and isolated compounds. These findings may be essential in future research with regard to black chokeberry based functional food products. Aronia melanocarpa is a component of food with a high biological potential.

\section{References}

1. Andrzej Sidor, Agnieszka Drożdżyńska, Anna Gramza-Michałowska (2019) Black chokeberry (Aronia melanocarpa) and its products as potential health-promoting factors - An overview. Trends in Food Science \& Technology 89: 45-60.

2. Petko N Denev, Christo G Kratchanov, Milan Ciz, Antonin Lojek, Maria G Kratchanova, et al. (2012) Bioavailability and Antioxidant Activity of Black Chokeberry (Aronia melanocarpa) Polyphenols: in vitro and in vivo Evidences and Possible Mechanisms of Action: A Review. Comprehensive Reviews in Food Science and Food Safety 11(5): 471-489.

3. M Boncheva (2012) Curative effects of the fruits of Aronia melanocarpa. General medicine 14(3): 39-48.

4. C Benjamin Naman, Jie Li, Arvin Moser, Jeffery M Hendrycks, Chunhua Yuan et al. (2015) Computer-Assisted Structure Elucidation of Black Chokeberry (Aronia melanocarpa) Fruit Juice Isolates with a New Fused Pentacyclic Flavonoid Skeleton. Org Lett 17(12): 2988-2991.

5. Tunde Jurikova, Jiri Mlcek, Sona Skrovankova, Daniela Sumczynski, Jiri Sochor, et al. (2017) Fruits of Black Chokeberry Aronia melanocarpa in the Prevention of Chronic Diseases. Molecules 22(6): 944.

6. Hoda Muddasarul, Hemaiswarya Shanmugam, Doble Mukesh (2019) Role of Phenolic Phytochemicals in Diabetes Management. Springer ISBN 978-981-13-8997-9.

7. Simeonov SB, Botushanov NP, Karahanian EB, Pavlova MB, Husianitis HK, et al. (2002) Effects of Aronia melanocarpa juice as part of the dietary regimen in patients with diabetes mellitus. Folia Med (Plovdiv) 44(3): 20-23.

8. Badescu Magda, Badulescu Oana, Badescu Laurentiu, Ciocoiu, Manuela, et al. (2015) Effects of Sambucus nigra and Aronia melanocarpa extracts on immune system disorders within diabetes mellitus. Pharmaceutical Biology 53(4).

9. Jan Oszmiański, Sabina Lachowicz (2016) Effect of the Production of Dried Fruits and Juice from Chokeberry (Aronia melanocarpa L) on the Content and Antioxidative Activity of Bioactive Compounds. Molecules 21(8): 1098-1105.

10. Miyuki Kozukaa, Takuya Yamane, Yoshihisa Nakan, Takenori Nakagaki, Iwaro Ohkubo, et al. (2015) Identification and characterization of a di- 
peptidyl peptidase IV inhibitor from aronia juice. Biochemical and Biophysical Research Communications 465(3): 433-436.

11. Takuya Yamane, Miyuki Kozuka, Momoko Imai, Yoshio Yamamoto, Iwao Ohkubo, et al. (2017) Reduction of blood pressure by aronia berries through inhibition of angiotensin-converting enzyme activity in the spontaneously hypertensive rat kidney. FFHD 7(4): 280-290.

12. Takuya Yamane, Miyuki Kozuka, Yoshio Yamamoto, Yoshihisa Nakano, Takenori Nakagaki, et al. (2016) Effectiveness of aronia berries for reduction of mild fibrosis and gene expression analysis in liver from mice fed a high-fat diet with aronia berries. FFHD 6(3): 144-157.

13. M Boncheva, G Georgiev, V Shishkov (2013) Intake of aronia melanocarpa juice improves medical tests and the feeling of health in patients with non-alcoholic fatty liver disease. General medicine 15(2): 21-30.

14. Takuya Yamane, Sato Murao, Miyuki Kozuka, Mari Shimizu, Junko Suzuki, et al. (2014) Serum DJ-1 level is positively associated with improvements in some aspects of metabolic syndrome in Japanese women through lifestyle intervention. Nutrition research 34(10): 851-855.

15. Kozuka M, Yamane T, Nakano Y, Nakagaki T, Ohkubo I, et al. (2015) Identification and characterization of a dipeptidyl peptidase IV inhibitor from aronia juice. Biochem Biophys Res Commun 465(3): 433-436.

16. Yamane T, Kozuka M, Konda D, Nakano Y, Nakagaki T, et al. (2016) Improvement of blood glucose levels and obesity in mice given aronia juice by inhibition of dipeptidyl peptidase IV and $\alpha$-glucosidase. J Nutr Biochem 31: 106-112.

17. Tjelle TE, Holtung L, Bøhn SK, Aaby K, Thoresen M, et al. (2015) Polyphenol-rich juices reduce blood pressure measures i $n$ a randomised controlled trial in high normal and hypertensive volunteers. Br J Nutr 114(7): 1054-1063.
18. Guerrero L, Castillo J, Quiñones M, Santiago Garcia-Vallvé, Arola L, et al. (2012) Inhibition of angiotensin-converting enzyme activity by flavonoids: structure -activity relationship studies. PLoS One 7(11): e49493.

19. M Boncheva, V Madjova, H Madjova (2018) Studying the effects of intake of aronia melanocarpa fresh fruit juice on carbohydrate exchange in patients with type 2 diabetes mellitus. General medicine 11(1): 27-33.

20. Margaritka Boncheva, Christiana Madjova, Valentina Madjova (2018) Investigating the effect of aronia melanocarpa fresh fruit juice intake on changes in laboratory hematology parameters in adolescents with obesity and diabetes mellitus. Journal Child \& Infectious Diseases 10(1): $9-14$

21. Margaritka Boncheva, Christiana Madjova, Valentina Madjova (2017) Investigating the effect of aronia melanocarpa fresh fruit juice intake on changes in laboratory parameters for oxidative stress in patients with type 2 diabetes mellitus. BMJ 11(3): 52-60.

22. Margaritka Boncheva, Christiana Madjova, Valentina Madjova (2018) Changes in nitrites oxidation of saliva in continuous intake of fresh fruit juice of aronia melanocarpa in adolescents with obesity and diabetes mellitus. Journal “CHILD \& INFECTIOUS DISEASES” 10(1): 2-8.

23. Boncheva, Christiana Madjova, Valentina Madjova (2018) Studying the effects of intake of aronia melanocarpa fresh fruit juice on plasma lipids exchange in patients with type 2 diabetes mellitus. BMJ 12(1): 34-37.

24. Kanti Bhooshan Pandey, Syed Ibrahim Rizvi (2009) Plant polyphenols as dietary antioxidants in human health and disease. Oxidative Medicine and Cellular Longevity 2(5): 270-278. 